\title{
Non-Perturbative High-Energy QCD
}

\section{Arthur Hebecker*}

Theory Division, CERN, CH-1211 Geneva 23, Switzerland

E-mail: 'arthur.hebecker@cern.chi

ABSTRACT: It is the aim of this talk to review our understanding of the high-energy limit of QCD, focussing, in particular, on recent theoretical developments. After a brief introduction, I will recall why the true high-energy limit of QCD scattering processes is genuinly non-perturbative and why it has so far not been possible to apply lattice methods to this type of physics. Given the experimental fact of slowly rising hadronic cross sections, we are thus faced with a fundamental problem comparable to that of confinement but without the promise of the lattice. During the last years, the experimental side of this field has largely been driven by the HERA accelerator, which has, naturally, also influenced recent theoretical work in high-energy QCD. I will therefore devote the second part of the talk to small- $x$ deep inelastic scattering, in particular the physics of diffraction, and attempt to describe its impact on the wider field of non-perturbative high-energy QCD.

\section{Introduction}

On the basis of an overwhelming amount of experimental data and its quantitative description by various theoretical methods, we can confidently say that quantum chromodynamics (QCD) is the correct theory of strong interactions. Structurally, this theory is extremely simple. It is defined by the gauge group $\mathrm{SU}(3)$ and the presence of a certain number of quark fields $\psi$ in the fundamental representation,

$$
\mathcal{L}=-\frac{1}{2} \operatorname{tr} F_{\mu \nu} F^{\mu \nu}+i \bar{\psi}(i \not D-m) \psi .
$$

The coupling constant $\alpha_{s}=g^{2} /(4 \pi)$ enters the above lagrangian via $D_{\mu}=\partial_{\mu}+i g A_{\mu}$ and $F_{\mu \nu}=(1 / i g)\left[D_{\mu}, D_{\nu}\right]$. The renormalization group teaches us that $\alpha_{s}$ is small in shortdistance (i.e., high-virtuality) processes and grows to large values in calculations relevant to long-distance phenomena (see, e.g., [1]). Thus, while the first type of processes can be controlled in perturbation theory, genuinely non-perturbative methods are needed to describe the physics of hadrons and low-energy interactions. QCD is therefore arguably the best-defined and at the same time most interesting example of a quantum field theory

\footnotetext{
${ }^{*}$ Speaker.
} 
that we know. The latter statement refers to the physics of strong interactions and the effect of confinement, which govern the low-energy domain. For a large and constantly growing number of low-energy observables, numerical simulations on the lattice, i.e., in a discretised euclidean version of the theory, provide a first-principles quantitative method of calculation (see, e.g., [2]).

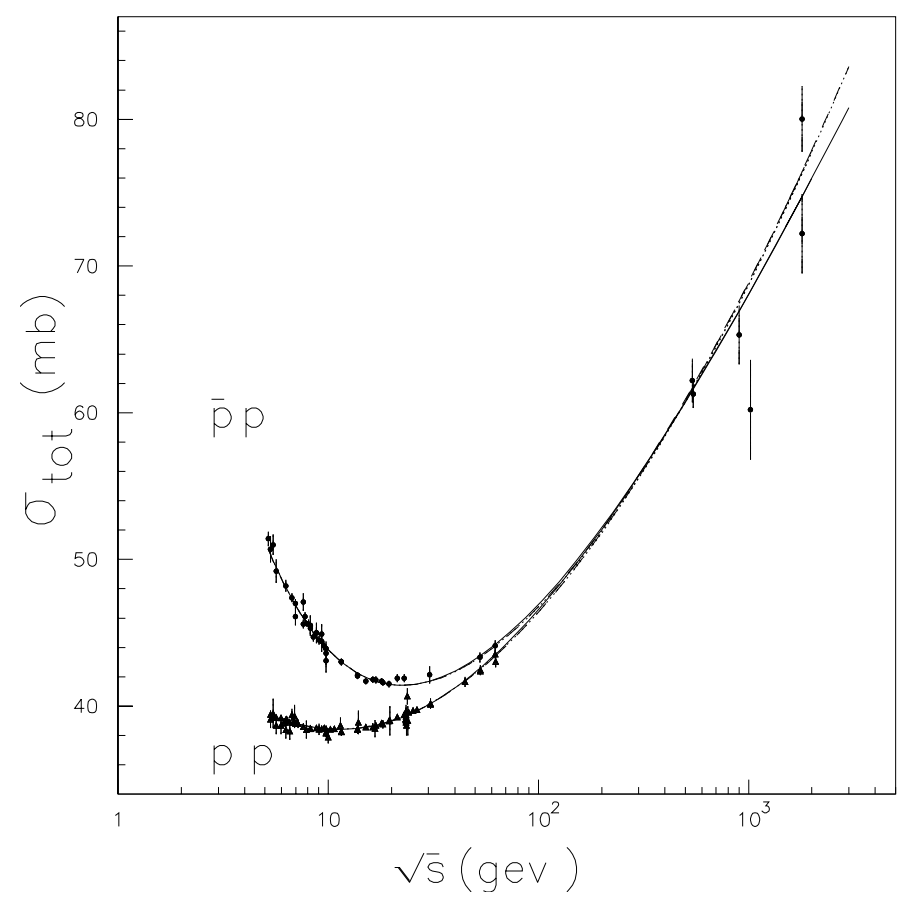

Figure 1: Total cross sections (from Ref. [3]).

However, there exists a qualitatively distinct class of observables, namely scattering processes with large center-of-mass energies but without large virtualities in intermediate states, where both perturbation theory and conventional euclidean lattice methods fail. These processes are the focus of the present review. Maybe the most prominent representative of these processes are total hadronic cross sections in the limit $s \rightarrow \infty$. The most interesting qualitative property of these cross sections is their slow rise with $s$, which is illustrated in Fig. 吕. The two cross sections ( $p p$ and $p \bar{p}$ ) can be parameterized by $s^{\delta}$ (here $\delta=$ $0.07), \ln s$ and $\ln ^{2} s$ at high energy. These parameterizations are at present indistinguishable on the basis of the data. It is surprising and highly unsatisfactory that we have no genuine understanding of the striking and universal phenomenon of asymptotically rising cross sections on the basis of the QCD lagrangian.

\section{Theoretical ideas concerning the asymptotic rise of cross sections}

\subsection{Geometrical picture}

Let us start with the simple observation that the high-energy total cross section of two hadrons (i.e., two complex extended objects) should, naively, be constant. They will simply always interact if they overlap in impact parameter space.

It is amusing to note that a very simple quantum-mechanical extension of this naive picture was given by Heisenberg as early as 1952 [4]. He assumed that the target hadron is surrounded by a field with energy density $\sim e^{-m_{\pi} r}$ (motivated by the Yukawa potential). Furthermore he conjectured that for an inelastic process to occur, the projectile has to pass so close to the target that there is locally enough energy in the collision of projectile and target field to create a pion pair. As the projectile energy grows, this effective maximal impact parameter grows as well, and one finds $\sigma \sim\left(1 / m_{\pi}^{2}\right) \ln ^{2}\left(s / m_{\pi}^{2}\right)$ for the total cross 
section. However, I am not aware of any quantum field theoretic version of the above argument.

Later it was shown on the basis of very general principles, such as unitarity and analyticity, that cross sections can not grow faster than $\ln ^{2}\left(s / s_{0}\right)$ in the limit $s \rightarrow \infty[5]$ (Froissart bound). However, in contrast to Heisenberg's simple argument, the derivation of this rigorous results does not provide a physical mechanism realising this growth. Thus, there is at present no clear field theoretic understanding of the transverse 'expansion' of hadrons at high energies.

\subsection{Regge theory}

Let us recall the basic underlying concepts of Regge theory, which, although not linked directly to the QCD lagrangian, provides a well-defined framework for the discussion of high-energy cross sections (see, e.g., $[6,7]$ ).

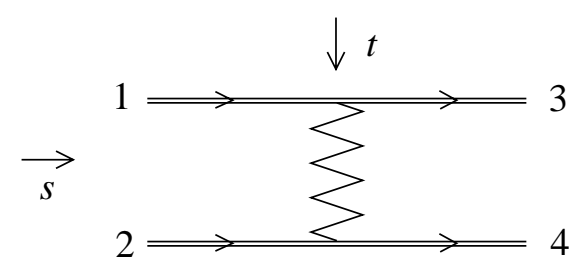

Figure 2: Scattering process $12 \rightarrow$ 34 via reggeon exchange.

Using analyticity and crossing symmetry, the amplitude $T_{12 \rightarrow 34}(s, t)$, depicted in Fig. $2 \overline{4}$, can be related to the amplitude $T_{1 \overline{3} \rightarrow \overline{2} 4}\left(s^{\prime}, t^{\prime}\right)$, where $s^{\prime}=t, t^{\prime}=s$, and barred numbers denote antiparticles. The partial wave expansion for this crossed amplitude reads

$$
T_{1 \overline{3} \rightarrow \overline{2} 4}\left(s^{\prime}, t^{\prime}\right)=\sum_{l=0}^{\infty}(2 l+1) a_{l}\left(s^{\prime}\right) P_{l}(\cos \theta),
$$

where $\theta$ is the centre-of-mass frame scattering angle, which is a function of $s^{\prime}, t^{\prime}$ and the particle masses, and $P_{l}$ are Legendre polynomials. Let the two functions $a_{\eta}(l, t)$ with $\eta=+1$ and $\eta=-1$ be the analytic continuations to complex $l$ of the two sequences $\left\{a_{l}(t), l=0,2,4, \ldots\right\}$ and $\left\{a_{l}(t), l=1,3,5, \ldots\right\}$. In the simplest nontrivial case, the only singularity of $a_{\eta}(l, t)$ is a single $t$-dependent pole at $l=\alpha(t)$. It can then be shown that, in the limit $s \rightarrow \infty$,

$$
T_{12 \rightarrow 34}(s, t)=\beta_{13}(t) \beta_{24}(t) \zeta_{\eta}(\alpha(t))\left(\frac{s}{s_{0}}\right)^{\alpha(t)}
$$

where $s_{0}$ is an arbitrary scale factor, $\beta_{13}$ and $\beta_{24}$ are two unknown functions of $t$, and

$$
\zeta_{\eta}(\alpha(t))=\frac{1+\eta e^{-i \pi \alpha(t)}}{\sin \pi \alpha(t)}
$$

is the signature factor, depending on the signature $\eta$ of the relevant Regge trajectory $\alpha(t)$. If $a_{\eta}(l, t)$ has a more complicated analytic structure, the rightmost singularity in the $l$ plane dominates the behaviour at large $s$.

Within the present context, the essential predictions of the asymptotic expression Eq. $\left(\overline{2} . \overline{2}_{2}\right)$ are the power-like energy dependence $s^{\alpha(t)}$ and the factorization of the two vertex factors $\beta_{13}(t)$ and $\beta_{24}(t)$. This last feature, which underlies the graphic representation of reggeon exchange in Fig. $\stackrel{2}{u}_{n}$, is relevant if the same Regge trajectory governs different scattering processes. Note also that, for positive $t=s^{\prime}$ and integer $l, \alpha(t)$ describes the positions of poles of the physical amplitude $T_{1 \overline{3} \rightarrow \overline{2} 4}\left(s^{\prime}, t^{\prime}\right)$. Such poles are expected whenever 
an on-shell particle with appropriate mass $m^{2}=s^{\prime}$ and angular momentum $l$ can be created in the collision of 1 and $\overline{3}$. Indeed, most Regge trajectories pass through known physical states with mass $m^{2}=t$ and angular momentum $\alpha(t)$.

The Froissart bound implies that $\alpha(0) \leq 1$ for all Regge trajectories. However, it was observed early on that a very good fit to $p p$ and $p \bar{p}$ cross sections could be obtained assuming the dominance of a single pole with $\alpha(0)>1$. The corresponding trajectory is known as the pomeron trajectory. Donnachie and Landshoff found that a large set of different hadronic cross sections can be fitted with an intercept $\alpha(0)=1.08$ [8]. In spite of the power-like growth of Eq. (י.2. Froissart bound is not violated below the Planck scale. It is then argued that unitarity is not a serious problem at all realistic energies. However, one should keep in mind that analyses based on a pomeron trajectory with $\alpha(0)>1$ are, strictly speaking, not selfconsistent in the framework of Regge theory. Therefore, it is likely that the rightmost singularity in the complex $l$ plane is not a single pole but a cut, in which case many of the results obtained in this framework are called into doubt.

In connection with the observed strong rise of $\gamma^{*} p$ cross sections at HERA, there has recently been a lot of discussion of the possible need for a 'second pomeron pole' [9] (see [10] for an update). However, as will be discussed below, this effect can also be understood in QCD perturbation theory.

\subsection{Ideas in euclidean field theory}

As already mentioned, the best developed method for addressing non-perturbative problems in non-abelian gauge theories is the lattice. The numerical simulation of the path integral forces one to work in euclidean field theory and to derive Minkowski-space observables by analytic continuation. There are well-known methods for the hadron spectrum, certain decay processes, and for operators relevant to deep inelastic scattering (DIS), but without the possibility to take the limit $x_{\mathrm{Bj}} \rightarrow 0$. However, no established procedure exists for the large- $s$ limit of hadronic cross sections. The fundamental difficulty becomes apparent by observing that the interesting dynamics resides in the soft fluctuations of the hadronic wave function, which, at $s \rightarrow \infty$ are localized on the light cone.

An interesting idea to overcome this problem was put forward some time ago by Meggiolaro [11] (see [12] for recent results). The approach rests on the well-known relation between the high-energy scattering of hadrons and correlators of light-like Wilson lines (which correspond to the trajectories of the constituent quarks) [13]. Meggiolaro showed that the expectation value of two Wilson lines forming a certain hyperbolic angle in Minkowski space and the expectation value of two Wilson lines forming a certain angle in euclidean space are connected by analytic continuation in the angular variables. One may now hope to describe the limit of light-like minkowskian Wilson lines (i.e., the physical limit $s \rightarrow \infty$ ) on the basis of a lattice calculation of Wilson line correlators in the euclidean theory. Note also that a conceptually related but different euclidean approach to the small- $x$ limit of DIS was suggested in [14].

Of course, in the above proposal it is still not clear how to technically obtain the dependence on an angular variable with a precision that is high enough for analytic con- 


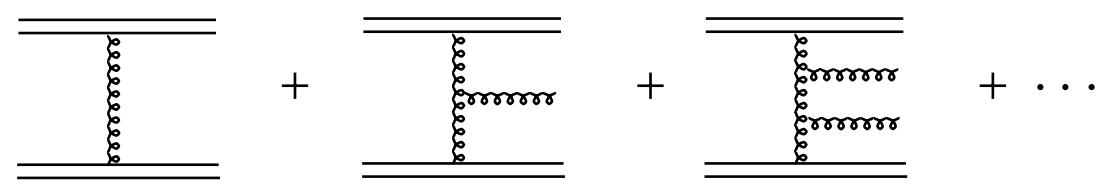

Figure 3: Two dipoles scattering via one-gluon exchange. Gluons can be radiated into the final state.

tinuation. The fundamental difficulty of this is obvious since the lattice breaks rotation invariance. This difficulty may be deeply related to the second fundamental problem of lattice approaches to high-energy observables, namely, the vast discrepancy of the two scales $\Lambda_{Q C D}$ and $s$. Such two-scale problems are difficult to approach since, on the lattice, one is confined to the region of scales between lattice spacing and the size of the simulated box.

A further new approach to the high-energy limit of QCD was proposed by Janik and Peschanski [15] (see also [16]). The authors suggest using the AdS/CFT correspondence (also known as the Maldacena conjecture) [17] to investigate high-energy scattering in nonabelian gauge theories. The AdS/CFT correspondence claims the equivalence of weakly coupled string theory in an Anti-de-Sitter (AdS) geometry with strongly coupled $\mathcal{N}=4$ super Yang-Mills theory, which is a conformal field theory (CFT), in 4-dimensional Minkowski space. Further, to make the connection to the realistic case of confining gauge theories, the authors use Witten's proposal [18] that a confining gauge theory is dual to string theory in an AdS black hole background. As discussed above, the high-energy scattering of two dipoles can be calculated from the correlation function of two Wilson loops in the euclidean theory. Using AdS/CFT correspondence, the calculation of the latter can be reduced to a minimal surface problem in an AdS black hole background.

\subsection{The BFKL approach}

In brief, one could say that the BFKL (Balitsky-Fadin-Kuraev-Lipatov) method attempts to approach the rise of hadronic cross sections from the perturbative side, by summing $\ln s$ enhancements appearing in higher orders of perturbation theory. Even though the technical realization is rather involved [19] (see [7] for a modern introductory text, [20] for results at next-to-leading order, and [21] for recent reviews), the main physical idea is simple. Consider the scattering of two small (perturbative) dipoles (Fig. 站). At leading order, the total cross section is determined by one-gluon exchange. At next-to-leading order, a gluon can be radiated into the final state. The phase space open to this gluon is limited by the rapidities of the two colliding dipoles and grows with $s$. This leads to a $\ln s$ enhancement of the total cross section. More final state gluons give rise to higher powers of $\ln s$. Keeping only the dominant terms, the whole series can be summed, giving rise to a cross section $\sigma \sim \alpha_{s}^{2} s^{\alpha_{B F K L}-1}$, where the 'BFKL intercept' is given by $\alpha_{B F K L}=1+12(\ln 2) \alpha_{s} / \pi$.

The $\ln s$ enhancement of higher orders in perturbation theory, which underlies this result, is of fundamental importance because it represents our only lagrangian-based derivation of a growing high-energy cross section. Nevertheless, the BFKL method in its present form is far from answering the fundamental question about the asymptotic high-energy behaviour. Firstly, it is not applicable to realistic hadrons because there is no hard scale 
justifying the perturbative method. Further, even if one restricts oneself to fictitious smalldipole ('onium-onium' - cf. [22]) collisions, BFKL does not provide the answer at $s \rightarrow \infty$. On the one hand, it clearly violates the Froissart bound. On the other hand, it violates unitarity at fixed impact parameter. This can be understood by interpreting the BFKL gluon radiation as a growing 'blackness' of the target, which is then probed by the projectile dipole. However, such an unlimited growth is impossible since the probability for the projectile to interact with the target at a given impact parameter eventually reaches unity.

On the technical side, the two above difficulties are related to the problems of higherorder corrections and of infrared diffusion (see, e.g. [23]). To understand the first problem, observe that even though BFKL sums terms $\sim \alpha_{s}^{2}\left(\alpha_{s} \ln s\right)^{n}$ for all $n$, one has all reason to expect the sum of contributions $\sim \alpha_{s}^{4}\left(\alpha_{s} \ln s\right)^{n}$ (e.g., 'double pomeron exchange') to grow faster as $s \rightarrow \infty$. More generally, one expects that even the extension of BFKL to any finite higher order will not provide the true high-energy asymptotics of the cross section. To understand the second problem, observe that the gluon ladder (the beginning of which is shown in Fig. (3i) 'knows' about the hard scale of the two scattering dipoles only via its ends. As $s$ increases, longer and longer gluon ladders become important and one sees numerically that the IR region starts to dominate the inner-rung momentum integrals.

Much of the recent work in BFKL physics has focussed on the proper understanding and implementation of the NLO corrections [20] which, naively, appeared to be extremely large, and on tests of BFKL dynamics at the available colliders. According to [24], the use of BLM scale setting improves the NLO situation dramatically. In a different approach, the improved small- $x$ evolution of [25], which incorporates the next-to-leading order BFKL kernel as well as renormalization group constraints on the relevant collinear limits, stabilizes the resummed results. For recent discussions of IR diffusion and of the relevancy of BFKL to the high-energy limit of DIS the reader is referred to refs. [26] and $[27,28]$. Other theoretical directions include the further study of reggeization (see, e.g. [29]) and the development of a reggeon field theory approach [30]. Being, at least in principle, very close to the ideal small-dipole case, the $\gamma^{*} \gamma^{*}$ cross section is an interesting testing field for BFKL methods. However, recent experimental data [31] and theoretical analyses [32] show that it is difficult to identify any direct evidence for BFKL dynamics in $\gamma^{*} \gamma^{*}$ collisions at presently available energies. Note furthermore that recently total cross section measurements for the collision of two real photons have been extended to very high energies and that a faster rise than expected on the basis of soft pomeron parameterizations has been observed [33].

Before closing this very brief section on BFKL, I would like to reiterate that most recent developments in this field are to be considered as work in perturbative QCD and that direct contact to the fundamental problem of the high-energy asymptotics is difficult to make. Therefore, in spite of its great interest in its own rights, the physics of BFKL lies somewhat outside the main line of development of this review.

\subsection{The method of high gluon densities}

The gluon ladder of BFKL (see Fig. $\hat{2}_{1}$ ) can be interpreted as the consecutive radiation of gluons by the target hadron, so that the growth of the cross section corresponds to a growth of the target gluon distribution with increasing $1 / x$. This small- $x$ enhancement 
of gluon distributions is also present in the more familiar DGLAP evolution of parton distributions [34]. As already mentioned before, the growth is limited by unitarity at any given point in impact space. The physical mechanism taming the growth is believed to be the recombination of partons [35]. Thus, the region of high gluon densities appears to be highly relevant to the question of how hadronic cross sections behave at very high energies.

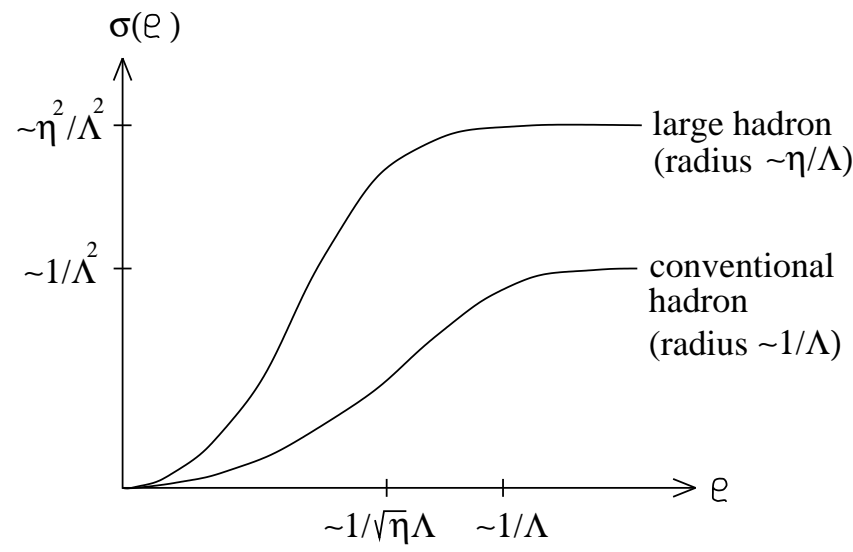

Figure 4: Qualitative behaviour of the dipole cross section $\sigma(\varrho)$ (here $\left.\Lambda \sim \Lambda_{Q C D}\right)$.
A lot of interest has been drawn to the the region of high gluon densities since the McLerranVenugopalan approach has offered the perspective of a new hardness scale and thus perturbative calculability in this region. The original proposal [36] dealt with large nuclei, where the new hardness scale can be understood by boosting the nucleus to very large energy and observing that the thickness of the target translates to a high density (i.e., small transverse separation) of glu-

ons in impact parameter space. This hardness scale can also be derived by considering small- $x$ DIS off a large nucleus in its rest frame [37] (cf. also [38,39]). In this approach, the total cross section is given by the convolution of the $\gamma^{*}$ wave function (characterizing the probability of a virtual photon to fluctuate into a $q \bar{q}$ pair of transverse size $\varrho$ ) and the dipole cross section $\sigma(\varrho)$ (characterizing the probability of this dipole to interact with the target). For conventional hadrons, even at high photon virtualities $Q^{2}$, dipole sizes up to $\sim 1 / \Lambda$ contribute (the region of the 'knee' in Fig. 'A' 1 ). For very large hadrons, one can see by purely geometrical arguments that the region of the knee is shifted to smaller values of $\varrho$, the relevant scale being $\sqrt{\eta} \Lambda$. Thus, the non-perturbative region is parametrically suppressed in the total cross section. Intuitively, this effect can be explained by saying that the largest dipoles that contribute are those for which saturation sets in, and that in large (thick) targets saturation sets in already for small dipoles.

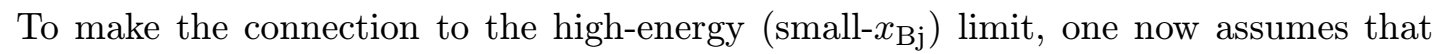
in the above arguments the thickness of the large target can be replaced by the extreme opacity that a usual hadronic target develops when probed by a very energetic projectile. In other words, since the knee in Fig. 'A- $\bar{A}_{1}^{-}$moves to the left in the small- $x_{\mathrm{Bj}}$ limit, saturation becomes a phenomenon that is, at least in principle, accessible in perturbation theory. Indeed, starting with $[38,40]$ (see [41] for earlier closely related work), over the last years impressive progress has been made in deriving and analysing the evolution of the gluon distribution within the McLerran-Venugopalan or high-density approach. In this framework, it is convenient to think of the hadron colour field as being characterized by the expectation value of Wilson lines penetrating the hadron. In the simplest case, these are the two Wilson lines corresponding to the $q \bar{q}$ pair into which the $\gamma^{*}$ fluctuates (cf. the quantity $\sigma(\varrho)$ above). To understand the energy dependence, one needs to consider an arbitrary 
number of such Wilson lines and to calculate the evolution of the generating functional for the corresponding expectation value in the target colour field. (Note that this can be understood as a renormalization group equation in which more and more energetic gluon field components are integrated out.) Even assuming the validity of perturbation theory throughout, the problem at hand is formidable.

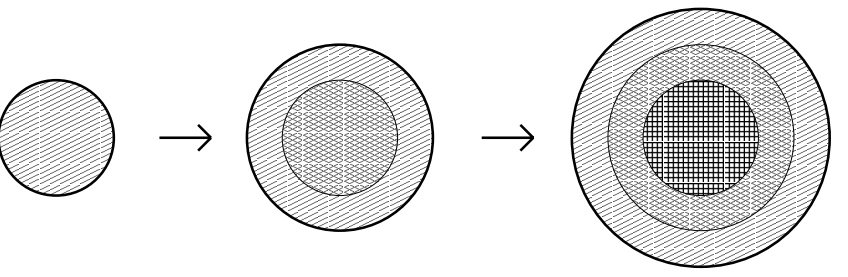

Figure 5: Qualitative picture of the high-energy evolution of a hadronic target in impact parameter space.

Recent work in the field is focussing on the full understanding of the above complicated non-linear evolution equation and on first attempts at physical applications $[42-$ 47]. In particular, the reader is referred to [42] for a comparative analysis of the methods employed in Refs. [40], [41], and [43]. Progress in solving the evolution equation using functional Focker-Planck methods has been reported in [44]. The application of the high gluon density regime, called 'colour glass condensate' in [45], to small- $x$ DIS has been discussed, e.g., in [46, 47].

However, one now has to ask how much closer the above developments bring us to a solution of the fundamental problem - the high-energy limit of hadronic cross sections. To discuss this question, consider the change of the impact parameter space picture of a hadron with increasing energy, Fig. parameter, the gluon density (or, equivalently, the interaction probability of an energetic small colour dipole) increases with energy. In Fig. $\overline{1}_{1}^{1}$, this is symbolized by increasing blackness. For given impact parameter and given dipole size, this growth is limited by unitarity. Technically, this is implemented by including non-linearities into the evolution equation - this is precisely the program of the McLerran-Venugopalan approach sketched above. Thus, one may indeed hope that this program will lead to a quantitative understanding of how, in the high energy limit, the target becomes completely black at any impact parameter. However, if total hadronic cross sections continue to grow asymptotically as $s \rightarrow \infty$, then this growth has to come from an effective transverse expansion in impact parameter space. As illustrated in Fig. edge of the disk, a region where gluon densities are not high and the applicability of the McLerran-Venugopalan method is not justified in any obvious way. In my opinion, it is this 'transverse' dynamics, i.e., the expansion of the target disk into the previously 'white' region, which represents the main challenge to the method of high gluon densities.

\section{Small- $x$ diffraction}

\subsection{Diffractive DIS as a tool to study high-energy QCD}

The small- $x$ limit of DIS (deep inelastic scattering) became experimentally viable only with the advent of the electron-(or positron-)proton collider HERA. The large centre-of-mass energy of the $e p$ collision, $\sqrt{s} \simeq 300 \mathrm{GeV}$, allows for a very high hadronic energy $W$ (the 

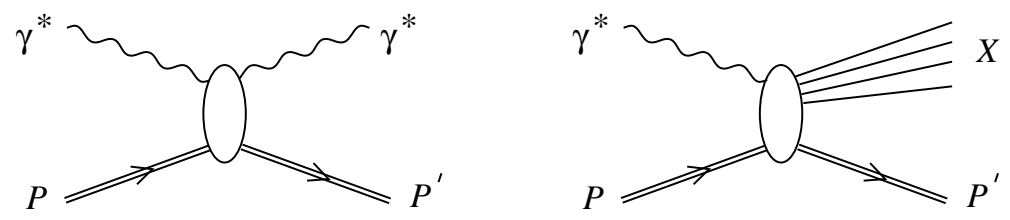

Figure 6: Forward Compton scattering and diffractive electroproduction.

cms energy of the $\gamma^{*} p$ collision) and thus for the observation of events with both very high photon virtuality $Q^{2}$ and very small $x_{\mathrm{Bj}}=Q^{2} /\left(Q^{2}+W^{2}\right)$.

Loosely speaking, diffraction is the subset of DIS characterized by a quasi-elastic interaction between virtual photon and proton. A particularly simple definition of diffraction is obtained by demanding that, in the $\gamma^{*} p$ collision, the proton is scattered elastically. Thus, in diffractive events, the final state contains the scattered proton with momentum $P^{\prime}$ and a diffractive hadronic state $X_{M}$ with mass $M$ (see the r.h. side of Fig. $\overline{\underline{6}}$ ).

Figure $\overline{6}_{i}^{\overline{6}}$ illustrates that diffractive DIS can be understood as a very special type of hadronic high-energy process. Inclusive small- $x$ DIS, i.e., the total high-energy $\gamma^{*} p$ cross section, is linked by the optical theorem to forward Compton scattering (1.h. side of Fig. '6i'). This is already a very interesting process since one has the additional variable $Q^{2}$ at one's disposal. In this sense, diffraction (r.h. side of Fig. ( $_{-1}^{-}$) is an even more interesting hadronic high-energy reaction since one now can also use the momentum transfer $t$ and the details of the hadronic final state $X$ to control the interaction between the two colliding objects. Given our ability to measure small- $x$ diffractive cross sections with high precision at the HERA accelerator, diffractive DIS can be used as a very powerful and versatile tool for the study of the high-energy behaviour of QCD, which significantly extends the physics capacity of hadron-hadron colliders. For recent reviews of diffractive DIS, the reader is referred to [48].

\subsection{The aligned jet model and its modern versions}

The aligned jet model [49] is based on a qualitative picture of DIS in the target rest frame, where the incoming virtual photon can be described as a superposition of partonic states. The large virtuality $Q^{2}$ sets the scale, so that states with low- $p_{\perp}$ partons, i.e., aligned configurations, are suppressed in the photon wave function. However, in contrast to high$p_{\perp}$ configurations, these aligned states have a large interaction cross section with the proton. Therefore, their contribution to DIS is expected to be of leading twist (of leading order in $\left.1 / Q^{2}\right)$. Since the above low- $p_{\perp}$ configurations represent transversely extended, hadron-like objects, which have a large elastic cross section with the proton, part of this leading twist contribution is diffractive. This very simple picture explains on a qualitative level the large cross section for diffractive or 'rapidity gap' events the observation of which caused significant excitement in the beginning of the HERA era [50].

The above intuitive picture was implemented in the framework of perturbative QCD in [51], where the perturbative fluctuation of the $\gamma^{*}$ into a $q \bar{q}$ pair was considered as the dominant process and the subsequent colour singlet exchange between the proton and the $q \bar{q}$ pair was realized by two gluons. A further essential step is the inclusion of higher Fock 
states in the photon wave function. In the framework of two gluon exchange, corresponding calculations for the $q \bar{q} g$ state were performed in [52]. The main shortcoming of the twogluon approach is the problem of justifying perturbation theory. As should be clear from the qualitative discussion of the aligned jet model, the diffractive kinematics is such that the $t$ channel colour singlet exchange does not feel the hard scale of the initial photon. Thus, more than two gluons can be exchanged without suppression by powers of $\alpha_{s}$.

This problem was systematically addressed in the semiclassical approach [53,54], where the interaction with the target was modelled as the scattering off a superposition of soft colour fields. In the high-energy limit, the eikonal approximation can be used to calculate the scattering of the energetic partons of the $\gamma^{*}$ fluctuation. Diffraction occurs if both the target and the partonic fluctuation of the photon remain in a colour singlet state. Thus, both the diffractive and inclusive DIS cross section can be calculated if a model for the wave functional of the proton is provided. A simple phenomenologically successful model for the proton colour field, which is based on the Glauber formula justified by the large-target approximation, was developed in [55].

Both the two-gluon-exchange and the semiclassical approach contain, as a first step, the perturbative calculation of the partonic $\gamma^{*}$ wave function, i.e., of the transition amplitude from the $\gamma^{*}$ to the $q \bar{q}$ or $q \bar{q} g$ state. In the simpler $q \bar{q}$ case, this wave function is then convoluted with the amplitude for the elastic interaction of the colour dipole and the target hadron. At $t=0$, this amplitude is determined by the dipole cross section $\sigma(\varrho)$ (cf. Sect. $2.5 \overline{2}$ ). Thus, one can now discuss different models for the target on the basis of $\sigma(\varrho)$. In particular, $\sigma(\varrho)$ can be modelled by perturbative two-gluon exchange with an ad-hoc IR cutoff or by the expectation value of two Wilson lines in the proton state (which is IR finite for a finite-size proton).

As far as $\sigma(\varrho)$ is concerned, certain relatively model-independent general statements can be made. The firmest one is probably the well-known relation to the inclusive gluon distribution $x g\left(x, \mu^{2}\right)[56]$,

$$
\sigma(\varrho)=\frac{\pi^{2}}{3} \alpha_{s}\left[x g\left(x, 1 / \varrho^{2}\right)\right] \varrho^{2}+\mathcal{O}\left(\varrho^{4}\right)
$$

valid at small $\varrho$. Note that this implies a dependence of $\sigma(\varrho)$ on the $x$ (or, equivalently, on the energy of the projectile $q \bar{q}$ pair) since $x g\left(x, \mu^{2}\right)$ is known to increase with $1 / x$ in the small- $x$ limit.

An intuitively obvious but quantitatively less clear statement is that this quadratic rise of $\sigma(\varrho)$ will eventually be tamed by non-perturbative effects, .i.e., a saturation of $\sigma(\varrho)$ has to occur at large $\varrho$. In the case of a very large target, this saturation sets in when $\varrho$ is still in the perturbative domain and a Glauber-type formula for $\sigma(\varrho)$ can be derived [55]. For a realistic hadron, the situation is less clear and the functional form of the saturation is unknown. Furthermore, one has to worry that saturation occurs at non-perturbative values of $\varrho$, where the dipole picture itself is questionable (see below).

A very simple parameterisation of $\sigma(\varrho)$, which takes into account the above generic 
features and which has been very popular recently, was suggested in [57]. It reads

$$
\sigma(\varrho, x)=\sigma_{0}\left\{1-\exp \left(-\frac{\varrho^{2}}{4 R_{0}^{2}(x)}\right)\right\}
$$

with $R_{0}=(1 / \mathrm{GeV})\left(x / x_{0}\right)^{\lambda / 2}$, i.e., it assumes a power-like growth of $x g\left(x, \mu^{2}\right)$ and a Glauber-type shape of saturation. The strength of this parameterisation lies in its simplicity and in the fact that it captures several important qualitative features of the dipole cross section. One shortcoming is that, in this parameterisation, the level at which $\sigma(\varrho)$ saturates is given by $\sigma_{0}$ and is therefore independent of the energy. This contradicts our experimental knowledge that soft hadronic cross sections grow with $s$. An interesting result of the application of the above parameterisation to diffractive and inclusive DIS is that both processes have a similar energy dependence. This remarkable scaling behaviour was previously pointed out in [58] and derived from very general considerations in the semiclassical framework in [53].

Unfortunately, there is one rather fundamental criticism that applies to all the above calculations based on the perturbative transition of the $\gamma^{*}$ to a set of partons and their subsequent interaction with the target proton. The problem is that the bulk of diffraction comes from partonic configurations, in particular $q \bar{q}$ pairs, which are not parametrically small on a scale of $\Lambda_{Q C D}$. Therefore, strictly speaking there is no reason to neglect non-perturbative gluonic interactions between quark and antiquark, and even worse, the interaction of the non-perturbative gluon field between the quarks with the gluon field of the target. To the best of my knowledge, the only case where this problem is under control is diffractive DIS of an optically very thick target described above. The required optical thickness can also arise in diffraction of protons at extremely high energies, but the applicability of this argument in the HERA domain is less than obvious.

\subsection{Diffractive parton distributions}

The conventional partonic interpretation of inclusive DIS appears to be most natural in the Breit frame (the frame where the photon momentum has the form $q=\left(0,0_{\perp}, Q\right)$ and the proton energy becomes very large as $x \rightarrow 0$ ). Viewing diffractive DIS in analogy to inclusive DIS, one arrives at a picture that is very different from the target rest frame picture of Sect. In this approach, the concept of fracture functions [59] or, more specifically, the diffractive parton distributions of [60] provide a framework firmly rooted in perturbative QCD. Recall that conventional parton distributions can be considered as probabilities for finding a parton with a certain momentum fraction in the fast moving proton. In short, diffractive parton distributions are conditional probabilities. A diffractive parton distribution $d f_{i}^{D}(y, \xi, t) / d \xi d t$ describes the probability of finding, in a fast moving proton, a parton $i$ with momentum fraction $y$, under the additional requirement that the proton remains intact while being scattered with invariant momentum transfer $t$ and losing a small fraction $\xi=x_{\mathbb{P}}$ of its longitudinal momentum. Thus, the corresponding $\gamma^{*} p$ cross section can be written as [61]

$$
\frac{d \sigma\left(x, Q^{2}, \xi\right)^{\gamma^{*} p \rightarrow p^{\prime} X}}{d \xi}=\sum_{i} \int_{x}^{\xi} d y \hat{\sigma}\left(x, Q^{2}, y\right)^{\gamma^{*} i}\left(\frac{d f_{i}^{D}(y, \xi)}{d \xi}\right),
$$




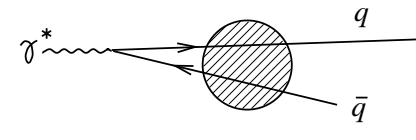

a)

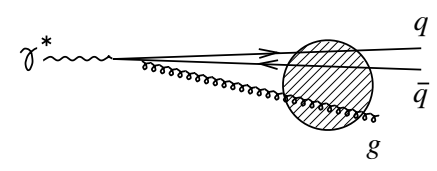

b)
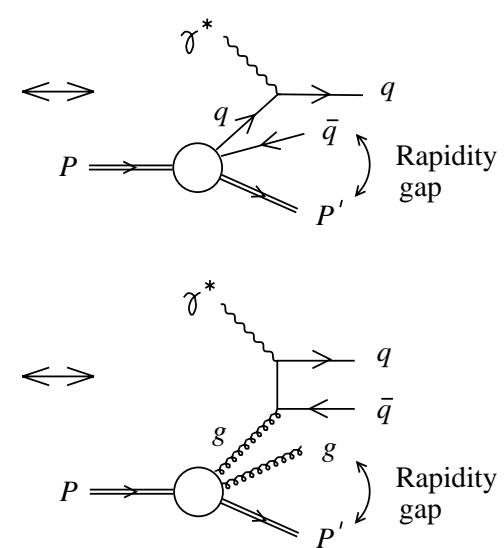

Figure 7: Diffractive DIS in the proton rest frame (left) and the Breit frame (right); asymmetric quark fluctuations correspond to diffractive quark scattering, asymmetric gluon fluctuations to diffractive boson-gluon fusion.

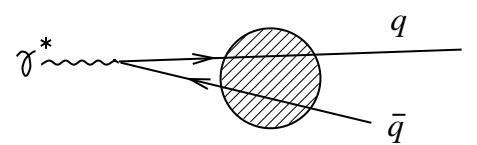

a)

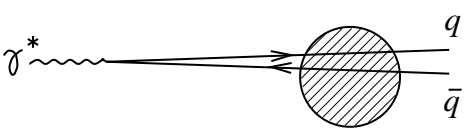

b)
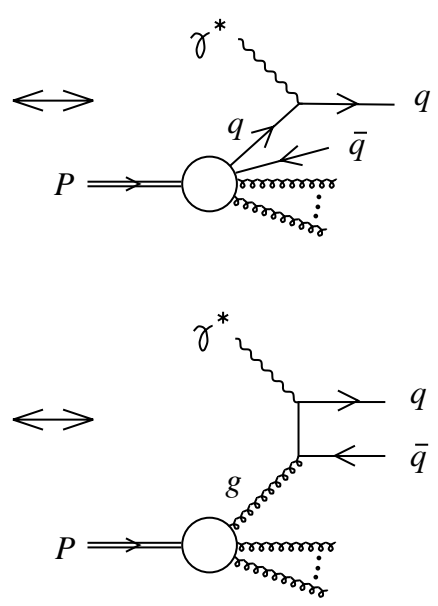

Figure 8: Inclusive DIS in the proton rest frame (left) and the Breit frame (right); asymmetric fluctuations correspond to quark scattering (a), symmetric fluctuations to boson-gluon fusion (b).

where $\hat{\sigma}\left(x, Q^{2}, y\right)^{\gamma^{*} i}$ is the total cross section for the scattering of a virtual photon characterized by $x$ and $Q^{2}$ and a parton of type $i$ carrying a fraction $y$ of the proton momentum. The above factorization formula holds in the limit $Q^{2} \rightarrow \infty$ with $x, \xi$ and $t$ fixed. Factorization proofs were given in [62] in the framework of a simple scalar model and in [63] in full QCD.

As in inclusive DIS, there are infrared divergences in the partonic cross sections and ultraviolet divergences in the parton distributions. Thus, a dependence on the factorization scale $\mu$ appears both in the parton distributions and in the partonic cross sections. The claim that Eq. (13.3) holds to all orders implies that these $\mu$ dependences cancel, as is well known in the case of conventional parton distributions. Therefore, the diffractive distributions obey the usual DGLAP evolution equations [61,64].

The connection of the above picture of diffractive and inclusive DIS with the previously discussed aligned jet or target rest frame picture is illustrated in Figs. 
example, the diffractive quark distribution (Fig. The leading twist contribution arises from fluctuations where one of the two quarks carries only a small longitudinal momentum fraction of the $\gamma^{*}$. Boosting to the Breit frame, one finds that this less energetic antiquark can be interpreted as a quark coming from the diffractive quark distribution of the proton. Putting this and the other subprocesses in Figs. $\bar{T}_{1}$ and ${ }_{-1}^{18}$ into equations, one finds that the diffractive and inclusive parton distributions can be expressed in terms of expectation values of Wilson loops in the proton colour field (or, similarly, in terms of the colour dipole cross sections $\sigma(\varrho))[55,65]$. One can then perform phenomenological analyses of inclusive and diffractive DIS on the basis of a model for the proton at some low scale $Q_{0}^{2}$ using conventional DGLAP evolution to make predictions at all $Q^{2} \geq Q_{0}^{2}[55,66,67]$.

Historically, the concept of diffractive parton distributions has a predecessor in the partonic interpretation of the pomeron [68]. In this approach, the quasi-elastic high-energy scattering of photon fluctuation and proton is interpreted in terms of pomeron exchange and it is assumed that the pomeron can, like a real hadron, be characterized by a parton distribution. This distribution is assumed to factorize from the pomeron trajectory and the pomeron-proton-proton vertex, which are both obtained from the analysis of purely soft hadronic reactions. The problem with this approach is the lacking justification of the pomeron idea and the factorization assumption in QCD. Furthermore, the observed energy dependence of diffractive DIS disagrees with the universal soft pomeron expectation, and the universality between diffraction in DIS and in hadron-hadron collisions, which is expected in this approach, is not observed [69]. As reported at this conference [70], a unified description of diffractive DIS and hadronic diffraction at the Tevatron can be achieved in Monte-Carlo models based on soft colour exchange [71]. For other approaches to this difficult problem see, e.g., [72].

\subsection{Hard colour dipole exchange}

Diffractive processes where the $t$ channel colour singlet exchange is governed by a hard scale include the electroproduction of heavy vector mesons [73], electroproduction of light vector mesons in the case of longitudinal polarization [74] or at large $t$ [75], and virtual Compton scattering (the process $\gamma^{*} p \rightarrow \gamma p^{\prime}$ ) [76-78]. In the leading logarithmic approximation, the relevant two-gluon form factor of the proton can be related to the inclusive gluon distribution [73]. Accordingly, a very steep energy dependence of the cross section, which is now proportional to the square of the gluon distribution, is expected.

To go beyond leading logarithmic accuracy, the non-zero momentum transferred to the proton has to be taken into account. This requires the use of skewed parton distributions (see [76] and refs. therein), which were discussed in [77] within the present context. Although their scale dependence is predicted by well-known evolution equations, only limited information about the relevant input distributions is available (see, however, [79] for possibilities of predicting the non-forward from the forward distribution functions). For the application of an NLO analysis to virtual Compton scattering the reader is referred to [80] and refs. therein. 


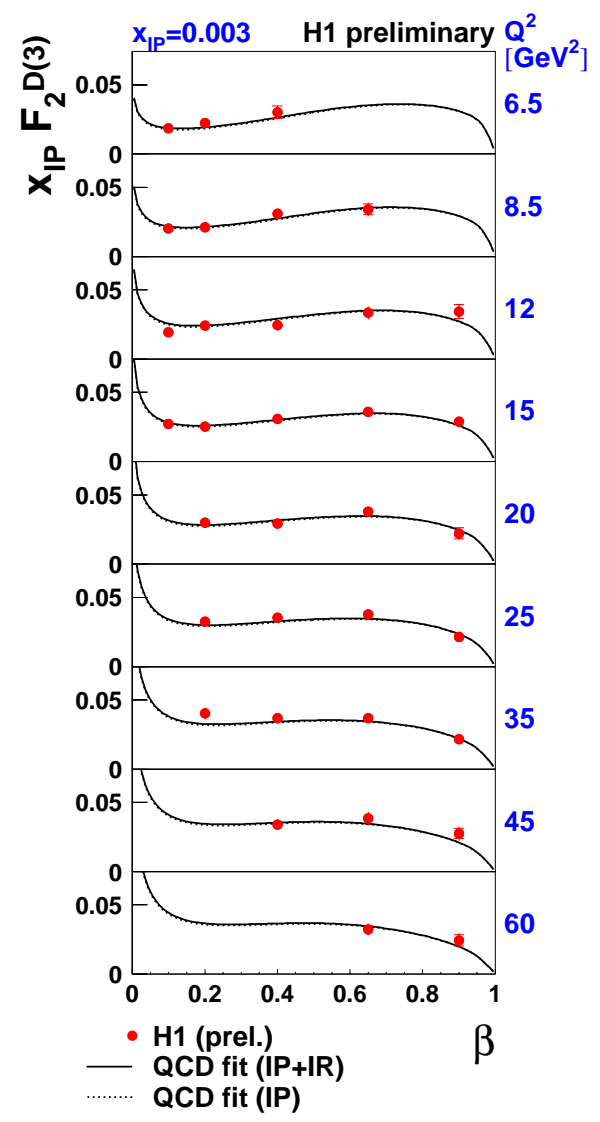

Figure 9: The measured diffractive structure function, plotted as $x_{\mathbb{P}} F_{2}^{D(3)}\left(\beta, Q^{2}, x_{\mathbb{P}}\right)$ (red data points), as a function of $\beta$ for various values of $Q^{2}$ and a fixed value $x_{\mathbb{P}}=$ 0.003. Also shown is the result of a QCD fit to the data as described in the text of conference paper 808 . The solid curves correspond to the sum of pomeron and leading reggeon exchange contributions, whereas the dotted curves indicate the contribution from pomeron exchange only. (Figure from H1 conference paper 808 [86]).
The perturbative calculations of meson electroproduction discussed above were put on a firmer theoretical basis by the factorization proof of [81]. Note, however, that the naive power counting expectation of the $Q^{2}$ dependence of cross sections is significantly modified by the anomalous dimension of the gluon distribution (cf. [82], where even the notoriously difficult ratio of longitudinal and transverse cross section is explained in a perturbative calculation). An interesting new field, closely related to exclusive vector meson production, are the semi-exclusive processes discussed in [83].

In connection with inclusive diffraction, it is interesting to point out that $F_{2}^{D(3)}\left(\beta, Q^{2}, \xi\right)$ at $\beta \rightarrow 1$ is dominated by hard colour singlet exchange and can therefore be calculated from the skewed gluon distribution [84] (see also [85]). Given the growing precision of the data, this perturbative character of $F_{2}^{D}$ at large $\beta$ will be essential for a full quantitative understanding of inclusive diffraction.

\subsection{New precision data from HERA}

The purpose of this section is to emphasize that new small- $x$ data from HERA is at present transforming diffractive DIS into a precision field. This transformation will be taken even further once the data from the HERA high-luminosity run starts to become available. As an illustration of the new quality of the data, consider the diffractive structure function measurement shown in Fig. $\overline{9}_{1}$ (for previous results see [87]). To understand this quantity, recall that, in addition to the conventional kinematic variables of DIS, $Q^{2}$ and $x_{\mathrm{Bj}}$, the diffractive process is characterized by $M$, the mass of the diffractive final state $X$. Alternatively, the variables $\beta=Q^{2} /\left(Q^{2}+M^{2}\right)$ or $\xi=x_{\mathbb{P}}=x / \beta$ can be used. Now, $F_{2}^{D(3)}\left(x, Q^{2}, \xi\right)$ is defined precisely as $F_{2}\left(x, Q^{2}\right)$, but on the basis of a cross section that is differential in $\xi$ as well as in $x$ and $Q^{2}$. Alternatively, one can replace $x$ by $\beta$ and write $F_{2}^{D(3)}\left(\beta, Q^{2}, \xi\right)$. The $Q^{2}$ evolution of the $\beta$ dependence, an essential ingredient of the method of diffractive parton distributions, is clearly visible in Fig. ${ }_{1-1}^{\bar{g}}$. Furthermore, the $\beta$ dependence is measured so well that one can now really hope to use diffraction as a tool to study the colour field of the proton. (Recall that, using the semiclassical framework, the 


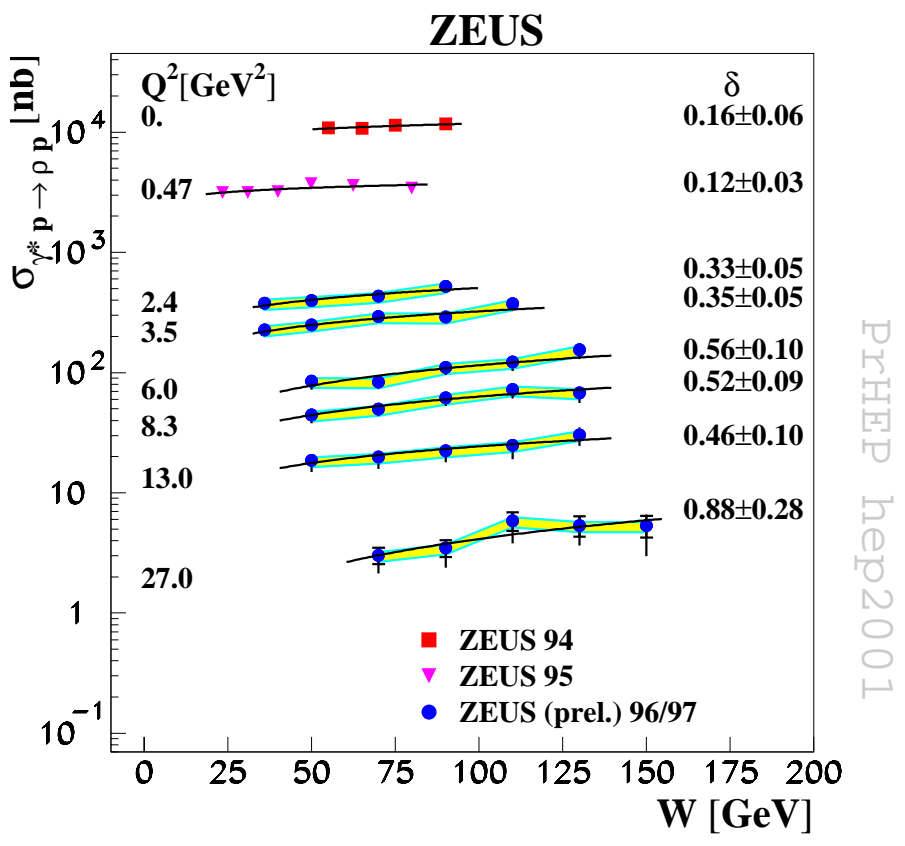

Figure 10: $W$ dependence of the cross section $\sigma\left(\gamma^{*} p \rightarrow \varrho^{0} p\right)$ for various $Q^{2}$ values as denoted in the figure. The data for $Q^{2}<1 \mathrm{GeV}^{2}$ obtained in previous ZEUS measurements [88] are shown for completeness. The dashed lines represent the results of fitting $\sigma \sim W^{\delta}$ at each $Q^{2}$ value and the results of the fit are shown in the figure. The shaded area indicates additional normalization uncertainties due to proton dissociation background (Figure from ZEUS conference paper 594 [89]).

$\beta$ dependence of the diffractive structure function is linked to the functional form of the expectation value of a Wilson loop in the proton state.)

Furthermore, as can be seen in Fig. $1 \overline{1} \underline{a}$, the energy dependence of vector meson electroproduction can now be measured in detail for different values of the photon virtuality $Q^{2}$. Since $Q^{2}$ is linked to the typical size of the $q \bar{q}$ dipole probing the proton field, one has yet another tool to study the energy dependence of the dipole cross section or, in other terms, the energy evolution of the effective proton colour field. Figure ${ }_{1}^{1} \overline{0} \overline{1}, 1$ shows the expected steeper energy dependence for small dipoles, where perturbative calculations are relevant. Note that it is an important long-term goal to experimentally verify that the fast growth of small-dipole cross sections softens above a certain energy (saturation in energy).

An interesting new result reported at the conference [90] concerns the odderon exchange (a gluonic $t$ channel exchange with $C=P=-1$ ). Given the observed suppression of the odderon in $p p$ and $p \bar{p}$ cross sections, it was suggested in [91] that the reason lies in the quark-diquark structure of the proton. If this is the case, then the diffractive production of pseudoscalar mesons at HERA with proton breakup in the final state should provide an ideal testing ground for the odderon. Employing a stochastic vacuum based approach 
in the description of the soft $t$-channel exchange [92], a prediction for, e.g., $\sigma_{\gamma p \rightarrow \pi^{0} X}$ was derived [91]. However, the measurements show no trace of this and similar odderon induced reactions [90] and thus the absence of the odderon remains mysterious.

In the future, it will be very interesting to see precision measurements of the diffractive structure function based on a tagged leading proton. First of all, such a measurement corresponds to the simplest and cleanest definition of diffractive DIS from the theoretical perspective. In particular, only in processes where the scattered proton is tagged can one be certain that the $\gamma^{*}$ partonic fluctuation was really probing the proton colour field and not some more complicated field corresponding to the transition between the proton and one of its excitations. Second, tagging the proton opens up the possibility of measuring the $t$ dependence of diffraction, i.e., $F_{2}^{D(4)}\left(\beta, Q^{2}, \xi, t\right)$, and this has the potential of resolving the transverse form of the target proton as seen at very high energy. This last possibility has already been exploited in [93] in the context of elastic meson production. Note that new and interesting diffractive measurements with tagged leading proton have been reported at this conference [94].

\section{Conclusions and outlook}

The high-energy limit of hadronic cross sections is a longstanding and fundamental problem in our understanding of the known interactions. In my opinion, it is at present not clear from which direction a solution might eventually emerge. There exist only few conceptually clean, lagrangian-based ideas of how to approach this problem. Among those are attempts of a translation of the problem to euclidean field theory, with the aim to perform a lattice calculation, as well as attempts to make use of the non-perturbative understanding of non-abelian gauge theories obtained in the framework of the AdS/CFT correspondence.

The only well-established QCD-based method of obtaining growing hadronic cross sections is, at present, the summation of $\ln s$ enhanced diagrams in perturbation theory. However, even though very impressive technical progress in this framework has been made over the past few years (NLO BFKL calculation, non-linear evolution equations at high gluon densities) it appears unlikely that the true high-energy asymptotics will become accessible with the methods available at present.

On the phenomenological side, the HERA accelerator is providing a rich and fruitful testing ground for theoretical ideas and methods. In particular, the measurement of diffractive and inclusive DIS allows for the study of hadronic reactions where one of the 'hadrons', the partonic fluctuation of the $\gamma^{*}$, can be tuned to one's needs. The most recent HERA results on small- $x$ physics have reached an impressive precision, and future results from the HERA high-luminosity run will transform diffractive high-energy scattering into a precision field. One may hope that, in the more distant future, the realisation of TESLA $\times$ HERA (THERA) will further expand the energy reach of the very promising, multifaceted reaction channel $\gamma^{*} p$ [95].

We look forward to the forthcoming precision data, the careful analysis of which we hope will provide essential clues to finding a first-principles theoretical approach to the complicated problem of the high-energy limit of QCD cross sections. 


\section{Acknowledgements}

I would like to thank the organizers of EPS-HEP 2001 for a very enjoyable and interesting conference and for their invitation to present this review.

\section{References}

[1] R.K. Ellis, W.J. Stirling and B.R. Webber, QCD and Collider Physics, Cambridge University Press, 1996.

[2] H. Wittig, plenary talk at EPS-HEP 99, Tampere, Finland, 1999 [hep-ph/9911400].

[3] P. Desgrolard, M. Giffon and E. Martynov, Nuovo Cim. A 110 (1997) 537 [hep-ph/9706306].

[4] W. Heisenberg, Z. Phys. 133 (1952) 65.

[5] M. Froissart, Phys. Rev. 123 (1961) 1053;

L. Łukaszuk and A. Martin, Nuovo Cim. A 52 (1967) 122.

[6] P.D.B. Collins, An Introduction to Regge Theory and High Energy Physics, Cambridge University Press, 1977.

[7] J.R. Forshaw and D.A. Ross, Quantum Chromodynamics and the Pomeron, Cambridge University Press, 1997.

[8] A. Donnachie and P.V. Landshoff, Nucl. Phys. B 244 (1984) 322 and Phys. Lett. B 296 (1992) 227 [hep-ph/9209205].

[9] A. Donnachie and P. V. Landshoff, Phys. Lett. B 437 (1998) 408 [hep-ph/9806344].

[10] P.V. Landshoff, talk at 9th Blois Workshop on Elastic and Diffractive Scattering, Prague, 2001 [hep-ph/0108156].

[11] E. Meggiolaro, Z. Phys. C 76 (1997) 523 [hep-th/9602104].

[12] E. Meggiolaro, hep-ph/0110069.

[13] O. Nachtmann, Ann. Phys. 209 (1991) 436.

[14] A. Hebecker, E. Meggiolaro and O. Nachtmann, Nucl. Phys. B 571 (2000) 26 [hep-ph/9909381].

[15] R.A. Janik and R. Peschanski, Nucl. Phys. B 549 (1999) 280 [hep-th/9907177] and 565 (2000) 193 [hep-th/0003059];

R.A. Janik, Phys. Lett. B 500 (2001) 118; [hep-th/0010069].

[16] C.-I. Tan, hep-ph/0102127.

[17] J. Maldacena, Adv. Theor. Math. Phys. 2 (1998) 231 [hep-th/9711200].

[18] E. Witten, Adv. Theor. Math. Phys. 2 (1998) 505 [hep-th/9803131].

[19] E.A. Kuraev, L.N. Lipatov and V.S. Fadin, Sov. Phys. JETP 44 (1976) 443 and 45 (1977) 199 ;

Y.Y. Balitsky and L.N. Lipatov, Sov. J. Nucl. Phys. 28 (1978) 822.

[20] V.S. Fadin and L.N. Lipatov, Phys. Lett. B 429 (1998) 127;

M. Ciafaloni and G. Camici, Phys. Lett. B 430 (1998) 349;

V. Del Duca and C.R. Schmidt, Phys. Rev. D 57 (1998) 4069 [hep-ph/9711309] and Phys. Rev. D 59 (1999) 074004 [hep-ph/9810215]. 
[21] G.P. Salam, Acta Phys. Polon. B 30 (1999) 3679 [hep-ph/9910492];

Carl R. Schmidt, talk at RADCOR 2000 [hep-ph/0106181].

[22] A.H. Mueller, Nucl. Phys. B 415 (1994) 373.

[23] J. Bartels, H. Lotter and M. Vogt, Phys. Lett. B 373 (1996) 215 [hep-ph/9511399];

J.R. Forshaw and P.J. Sutton, Eur. Phys. J. C 1 (1998) 285 [hep-ph/9703225];

J. Kwiecinski and L. Motyka, Phys. Lett. B 462 (1999) 203 [hep-ph/9905567];

K. Golec-Biernat, L. Motyka and A.M. Stasto, [hep-ph/0110325].

[24] S.J. Brodsky et al., JETP Lett. 70, 155 (1999) [hep-ph/9901229].

[25] M. Ciafaloni, D. Colferai and G.P. Salam, Phys. Rev. D 60 (1999) 114036 [hep-ph/9905566] and JHEP 9910 (1999) 017 [hep-ph/9907409].

[26] M. Ciafaloni, M. Taiuti and A.H. Mueller, Nucl. Phys. B 616 (2001) 349 [hep-ph/0107009].

[27] M. Ciafaloni, D. Colferai and G.P. Salam, JHEP 0007 (2000) 054 [hep-ph/0007240].

[28] G. Altarelli, R.D. Ball and S. Forte, hep-ph/0109178.

[29] C. Ewerz, JHEP 0104 (2001) 031 [hep-ph/0103260].

[30] L.N. Lipatov, Phys. Rept. 320 (1999) 249.

[31] L3 Collab., M. Acciarri et al., Phys. Lett. B 453 (1999) 333;

OPAL Collab., G. Abbiendi et al., hep-ex/0110006;

M. Przybycien, these proceedings.

[32] M. Cacciari, V. Del Duca, S. Frixione and Z. Trocsanyi, JHEP 0102 (2001) 029

[hep-ph/0011368];

V. Del Duca, these proceedings.

[33] L3 Collab., M. Acciarri et al., Phys. Lett. B 519 (2001) 33 [hep-ex/0102025].

[34] V.N. Gribov and L.N. Lipatov, Sov. J. Nucl. Phys. 15 (1972) 438, 675;

G. Altarelli and G. Parisi, Nucl. Phys. B 126 (1977) 298;

Yu.L. Dokshitzer, Sov. Phys. JETP 46 (1977) 641.

[35] L.V. Gribov, E.M. Levin and M.G. Ryskin, Phys. Rept. 100 (1983) 1.

[36] L. McLerran and R. Venugopalan, Phys. Rev. D 49 (1994) 2233 [hep-ph/9309289], D 49 (1994) 3352 [hep-ph/9311205], and D 50 (1994) 2225 [hep-ph/9402335].

[37] A. Hebecker and H. Weigert, Phys. Lett. B 432 (1998) 215.

[38] J. Jalilian-Marian, A. Kovner, L. McLerran and H. Weigert, Phys. Rev. D 55 (1997) 5414.

[39] Y.V. Kovchegov and A.H. Mueller, Nucl. Phys. B 529 (1998) 451 [hep-ph/9802440].

[40] J. Jalilian-Marian, A. Kovner, A. Leonidov and H. Weigert Nucl. Phys. B 504 (1997) 415 [hep-ph/9701284] and Phys. Rev. D 59 (1999) 014014 [hep-ph/9706377];

J. Jalilian-Marian, A. Kovner and H. Weigert, Phys. Rev. D 59 (1999) 014015

[hep-ph/9709432].

[41] I. Balitsky, Nucl. Phys. B 463 (1996) 99 [hep-ph/9509348].

[42] A. Kovner, J.G. Milhano and H. Weigert, Phys. Rev. D 62 (2000) 114005 [hep-ph/0004014].

[43] Y.V. Kovchegov, Phys. Rev. D 60 (1999) 034008 [hep-ph/9901281]. 
[44] H. Weigert, hep-ph/0004044.

[45] E. Iancu, A. Leonidov and L.D. McLerran, Nucl. Phys. A 692 (2001) 583 [hep-ph/0011241]; E. Ferreiro, E. Iancu, A. Leonidov and L. McLerran, hep-ph/0109115.

[46] I.I. Balitsky and A.V. Belitsky, hep-ph/0110158.

[47] A. Kovner and U.A. Wiedemann, Phys. Rev. D 64 (2001) 114002 [hep-ph/0106240].

[48] M. Wüsthoff and A.D. Martin, J. Phys. G 25 (1999) R309 [hep-ph/9909362];

A. Hebecker, Phys. Rept. 331 (2000) 1 [hep-ph/9905226];

J. Bartels and H. Kowalski, Eur. Phys. J. C 19 (2001) 693 [hep-ph/0010345].

[49] J.D. Bjorken and J.B. Kogut, Phys. Rev. D 8 (1973) 1341.

[50] ZEUS Collab., M. Derrick et al., Phys. Lett. B 315 (1993) 481;

H1 Collab., T. Ahmed et al., Nucl. Phys. B 429 (1994) 477.

[51] N.N. Nikolaev and B.G. Zakharov, Z. Phys. C 49 (1991) 607.

[52] N.N. Nikolaev and B.G. Zakharov, Z. Phys. C 64 (1994) 631 [hep-ph/9306230].

[53] W. Buchmüller and A. Hebecker, Nucl. Phys. B 476 (1996) 203 [hep-ph/9512329].

[54] W. Buchmüller, M.F. McDermott and A. Hebecker, Nucl. Phys. B 487 (1997) 283 [hep-ph/9607290].

[55] W. Buchmüller, T. Gehrmann and A. Hebecker, Nucl. Phys. B 537 (1999) 477 [hep-ph/9808454].

[56] L. Frankfurt, G.A. Miller and M. Strikman, Phys. Lett. B 304 (1993) 1 [hep-ph/9305228].

[57] K. Golec-Biernat and M. Wüsthoff, Phys. Rev. D 59 (1999) 014017 [hep-ph/9807513] and D 60 (1999) 114023 [hep-ph/9903358].

[58] W. Buchmüller, Phys. Lett. B 353 (1995) 335 [hep-ph/9504245].

[59] L. Trentadue and G. Veneziano, Phys. Lett. B 323 (1994) 201.

[60] A. Berera and D.E. Soper, Phys. Rev. D 50 (1994) 4328.

[61] A. Berera and D.E. Soper, Phys. Rev. D 53 (1996) 6162.

[62] M. Grazzini, L. Trentadue and G. Veneziano, Nucl. Phys. B 519 (1998) 394.

[63] J.C. Collins, Phys. Rev. D 57 (1998) 3051.

[64] J. Blümlein and D. Robaschik, Phys. Lett. B 517 (2001) 222 [hep-ph/0106037];

J. Blümlein, these proceedings.

[65] A. Hebecker, Nucl. Phys. B 505 (1997) 349 [hep-ph/9702373].

[66] F. Hautmann, Z. Kunszt and D.E. Soper, Nucl. Phys. B 563 (1999) 153 [hep-ph/9906284].

[67] K. Golec-Biernat and M. Wusthoff, Eur. Phys. J. C 20 (2001) 313 [hep-ph/0102093].

[68] G. Ingelman and P. Schlein, Phys. Lett. B 152 (1985) 256;

A. Donnachie and P.V. Landshoff, Phys. Lett. B 191 (1987) 309.

[69] L. Alvero et al., Phys. Rev. D 59 (1999) 074022 [hep-ph/9805268].

[70] R. Enberg, G. Ingelman and N. Timneanu, hep-ph/0106246;

$\mathrm{N}$. Timneanu, these proceedings. 
[71] A. Edin, G. Ingelman and J. Rathsman, Phys. Lett. B 366 (1996) 371 [hep-ph/9508386].

[72] E. Gotsman, E. Levin and U. Maor, Phys. Rev. D 60 (1999) 094011 [hep-ph/9902294];

A. B. Kaidalov, V. A. Khoze, A. D. Martin and M. G. Ryskin, Eur. Phys. J. C 21 (2001) 521 [hep-ph/0105145].

[73] M.G. Ryskin, Z. Phys. C 57 (1993) 89.

[74] S.J. Brodsky et al., Phys. Rev. D 50 (1994) 3134.

[75] J.R. Forshaw, M.G. Ryskin, Z. Phys. C 68 (1995) 137.

[76] D. Müller et al., Fortsch. Phys. 42 (1994) 101.

[77] X. Ji, Phys. Rev. Lett. 78 (1997) 610;

A.V.Radyushkin, Phys. Lett. B 380 (1996) 417.

[78] A.V. Belitsky and D. Müller, these proceedings, hep-ph/0111037.

[79] A.G. Shuvaev, K.J. Golec-Biernat, A.D. Martin and M.G. Ryskin, Phys. Rev. D 60 (1999) 014015 .

[80] A. Freund and M. F. McDermott, [hep-ph/0106319].

[81] J.C. Collins, L. Frankfurt and M. Strikman, Phys. Rev. D 56 (1997) 2982.

[82] A. D. Martin, M. G. Ryskin and T. Teubner, Phys. Rev. D 55 (1997) 4329 [hep-ph/9609448] and D 62 (2000) 014022 [hep-ph/9912551].

[83] S.J. Brodsky, M. Diehl, P. Hoyer and S. Peigne, Phys. Lett. B 449 (1999) 306 [hep-ph/9812277].

[84] A. Hebecker and T. Teubner, Phys. Lett. B 498 (2001) 16 [hep-ph/0010273].

[85] J. Bartels, J. R. Ellis, H. Kowalski and M. Wüsthoff, Eur. Phys. J. C 7 (1999) 443 [hep-ph/9803497].

[86] P. Newman, these proceedings.

[87] H1 Collab., C. Adloff et al., Z. Phys. C 76 (1997) 613;

ZEUS Collab., J. Breitweg et al., Eur. Phys. J. C 6 (1999) 43.

[88] ZEUS Collab., J. Breitweg et al., Eur. Phys. J. C 6 (1999) 603 [hep-ex/9808020] and C 2 (1998) 247 [hep-ex/9712020].

[89] A. Meyer, these proceedings;

B. Mellado, these proceedings.

[90] T. Golling, these proceedings (abstract 795).

[91] E.R. Berger et al., Eur. Phys. J. C 9 (1999) 491 [hep-ph/9901376] and C 14 (2000) 673 [hep-ph/0001270].

[92] H.G. Dosch and A. Krämer, Phys. Lett. B 252 (1990) 669.

[93] S. Munier, A.M. Stasto and A.H. Mueller, Nucl. Phys. B 603 (2001) 427 [hep-ph/0102291].

[94] F. Goebel, these proceedings (abstracts 566 and 809).

[95] TESLA-N Study Group Collab., H. Abramowicz et al., TESLA Technical design report, Pt. 6: Appendices. Chapter 2, DESY-01-011. 